\title{
Avaliação de IFN- $\gamma$ e IL-10 em cães naturalmente infectados com Leishmania (Leishmania) chagasi com e sem manifestações clínicas ${ }^{1}$
}

\author{
Carla J.R.M. Rosário ${ }^{2 *}$, Marina F. Dominici³ ${ }^{3}$ Maria S.C.O. Braga ${ }^{4}$, \\ Cristian A.A Lima ${ }^{3}$, José G. Pereira ${ }^{4}$ e Ferdinan A. Melo ${ }^{4}$
}

\begin{abstract}
Rosário C.J.R.M., Dominici M.F., Braga M.S.C.B., Lima C.A.A., Pereira J.G. \& Melo F.A. 2018. [Evaluation of INF- $\gamma$ and IL-10 in dogs naturally infected by Leishmania (Leishmania) chagasi with and without clinic events.] Avaliação de IFN- $\gamma$ e IL-10 em cães naturalmente infectados por Leishmania (Leishmania) chagasi com e sem manifestações clínicas. Pesquisa Veterinária Brasileira 38(4):722-725. Departamento de Patologia, Universidade Estadual do Maranhão, Cidade Universitária Paulo VI, Tirirical, São Luís, MA 65055-00, Brazil. E-mail: carlajanaina_rm@hotmail.com

Leishmaniasis has as obligatory intracellular parasitic etiological agents belonging to the genus Leishmania capable of infecting different species of mammals and reproducing them within the mononuclear phagocytic system. Domestic dogs are the main responsible for maintaining the epidemiological chain of the disease, presenting a wide variety of clinical profiles, from apparently healthy to severely affected. The expression of the cytokines from dogs naturally infected with Leishmania (Leishmania) chagasi was evaluated. Blood samples were collected from 50 animals, 20 from positive and symptomatic dogs for Leishmaniasis Canine (CVL), 20 from positive asymptomatic animals and 10 negative. Samples were analyzed by immunochromatographic test Dual Path Platform (DPP/Biomanguinhos ${ }^{\circledR}$ ) and by indirect ELISA (EIE/Biomanguinhos ${ }^{\circledR}$ ) for detection of anti-Leishmania antibodies. There was statistical difference between the groups observing an increase in IFN- $\gamma$ levels in asymptomatic animals and an IL-10 increase in symptomatic.
\end{abstract}

INDEX TERMS: INF- $\gamma$, IL-10, dogs, Leishmania (Leishmania) chagasi, leishmaniasis, clinical signs, immunology, parasitoses.

RESUMO.- As leishmanioses têm como agentes etiológicos parasitas intracelulares obrigatórios pertencentes ao gênero Leishmania capazes de infectar diferentes espécies de mamíferos e nestes se reproduzirem dentro do sistema fagocítico mononuclear. Os cães domésticos são os principais responsáveis pela manutenção da cadeia epidemiológica da doença, podendo apresentar uma grande variedade de

\footnotetext{
${ }^{1}$ Recebido em 9 de agosto de 2016.

Aceito para publicação em 5 de abril de 2017.

Pesquisa de Mestrado do primeiro autor financiada pela CAPES.

${ }^{2}$ Doutoranda pela Rede de Biodiversidade e Biotecnologia da Amazônia Legal (Bionorte), Universidade Estadual do Maranhão (UEMA), Cidade Universitária Paulo VI, Tirirical, São Luís, MA 65055-00, Brasil. *Autor para correspondência: carlajanaina_rm@hotmail.com

${ }^{3}$ Graduado(a) em Medicina Veterinária, Universidade Estadual do Maranhão (UEMA), Cidade Universitária Paulo VI, Tirirical, São Luís, MA 65055-00.

${ }^{4}$ Docente do Departamento de Patologia, Universidade Estadual do Maranhão (UEMA), Cidade Universitária Paulo VI, Tirirical, São Luís, MA 65055-00.
}

perfis clínicos, desde aparentemente sadios a severamente acometidos. Avaliou-se a expressão das citocinas de cães naturalmente infectados com Leishmania (Leishmania) chagasi. Foram coletadas 50 amostras, sendo 20 de animais positivos e sintomáticos para Leishmaniose Visceral Canina (LVC), 20 de animais positivos e assintomáticos e 10 de animais sabidamente negativos para a LVC. As amostras foram analisadas pelo teste imunocromatográfico rápido Dual Path Platform (DPP/Biomanguinhos ${ }^{\circledR}$ ) e pelo ELISA (EIE/Biomanguinhos ${ }^{\circledR}$ ) indireto para detecção de anticorpos anti-Leishmania. Após as confirmações dos testes, foi realizado o ELISA de captura (R \& D Systems) para quantificação das citocinas IL-10 e IFN- $\gamma$. Houve diferença estatística entre os grupos observando um aumento nos níveis de IFN- $\gamma$ nos animais assintomáticos e um aumento de IL-10 nos sintomáticos.

TERMOS DE INDEXAÇÃO: IFN- $\gamma$, IL-10, cães, Leishmania (Leishmania) chagasi, sinais clínicos, leishmaniose, imunologia, parasitoses. 


\section{INTRODUÇÃO}

As leishmanioses têm como agentes etiológicos parasitas intracelulares obrigatórios pertencentes ao gênero Leishmania capazes de infectar diferentes espécies de mamíferos e nestes se reproduzirem dentro do sistema fagocítico mononuclear (Gontijo \& Melo 2004).

Os cães domésticos são os principais responsáveis pela manutenção da cadeia epidemiológica da doença, pois vivem diretamente com o homem. Podendo apresentar uma grande variedade de perfis clínicos, desde aparentemente sadios a severamente acometidos, sendo este grau de apresentação dos sinais clínicos determinado pela cepa do parasito, genética e estado imunológico do animal (Handman \& Bullen 2002). Um fato intrigante é que a doença canina pode permanecer clinicamente inaparente por longos períodos e este é um dos principais problemas na erradicação da leishmaniose, por isso é de extrema importância o estudo imunológico dessa enfermidade.

Sendo o parasita obrigatoriamente intracelular o seu primeiro alvo são os fagócitos. Porém, o controle da infecção está associado à destruição intracelular dos amastigotas pelos macrófagos, pela ativação das células NK e pelos linfócitos T (Santos-Gomes et al. 2002).

Em camundongos, caracterizou-se que a resistência à infecção está associada a resposta Th1 com a produção de citocinas IL-12, IFN- $\gamma$ e TNF- $\alpha$, enquanto a suscetibilidade deve-se a resposta regulatória Th2 com a produção de IL-4, IL-10 e TGF- $\beta$. Porém é importante destacar que há diferença entre a progressão da enfermidade na infecção experimental ou natural já que a saliva do vetor tem função imunomodulatória (Paranhos et al. 1993).

Desta forma torna-se importante a avaliação da expressão de citocinas, na infecção natural por Leishmania (Leishmania) chagasi em cães com e sem manifestações clínicas.

\section{MATERIAL E MÉTODOS}

Amostras. Cinquenta cães independentes do sexo, raça e idade, foram selecionados aleatoriamente, dos atendimentos do Hospital Veterinário Universitário da Universidade Estadual do Maranhão (UEMA). Os cães foram submetidos ao exame clínico detalhado e os dados foram anotados em fichas clínicas individuais. Aqueles cães com infecção por Leishmania chagasi comprovada pelo teste rápido (DPP/Biomanguinhos ${ }^{\circledR}$ ), e pelo teste sorológico (EIE-LVC/Biomanguinhos ${ }^{\circledR}$ ), foram divididos em grupos com formas polares da doença: um grupo com vinte animais assintomáticos e outro com vinte animais com sinais bem definidos para LVC e um terceiro composto por 10 animais livres de infecção por L. chagasi.

O projeto foi aprovado pelo Comitê de Ética em Experimentação Animal da Universidade Estadual do Maranhão (CEEA/UEMA), processo n⿳0 020/2014. Amostras de sangue foram obtidas através da venopunção da veia cefálica ou jugular dos animais e acondicionadas em tubos de ensaio. Logo após o soro foi acondicionado em microtubo de polietileno e armazenado a $-20^{\circ} \mathrm{C}$ até a realização dos testes e avaliação do perfil de IL-10 e IFN- $\gamma$.

Sorologia para leishmaniose. O teste de ELISA (EIE-LVC/Biomanguinhos ${ }^{\circledR}$ ) indireto foi realizado com o objetivo de confirmar os animais positivos e negativos no DPP-Biomanguinhos ${ }^{\circledR}$, juntamente com a sintomatologia clínica dos cães, por serem oriundos de uma área endêmica e com um alto índice de transmissão. Identificando os que apresentavam título de anticorpos para
Leishmania (Leishmania) chagasi. Os cães que apresentaram títulos baixos associados à clínica e aos demais índices, foram classificados em animais livres da infecção por Leishmania (Leishmania) chagasi e utilizados no grupo controle. 0 ponto de corte considerado para o ensaio foi de 0,375 determinado pela média da densidade óptica dos orifícios do controle negativo multiplicado por dois. As microplacas de polietileno de 96 poços (EIE-LVC/Biomanguinhos ${ }^{\circledR}$ ) são previamente sensibilizadas com Leishmania major. Foram diluídos em tubos, $5 \mu \mathrm{L}$ dos controles e das amostras de soro homogeneizadas, em $500 \mu \mathrm{L}$ do diluente (1:100) disponibilizado no kit. Logo em seguida, na placa sensibilizada foram distribuídos $100 \mu \mathrm{L}$ dos controles e das amostras já diluídas. A placa foi levada à estufa à $37^{\circ} \mathrm{C}$ por 30 minutos. Logo após o conteúdo da placa foi lavado $6 x$ em seguida, com $200 \mu \mathrm{L} /$ orifício, aguardando 30 segundos entre cada lavagem. Foram homogeneizados e distribuídos $100 \mu \mathrm{L}$ da diluição do conjugado em cada poço da placa e incubada novamente a $37^{\circ} \mathrm{C}$ por $30 \mathrm{~min}$. Lavados novamente em solução PBS para então serem distribuídos $100 \mu \mathrm{L}$ do substrato em todos os orifícios. Deixado a temperatura ambiente e ao abrigo da luz, durante 30 minutos. Em seguida, foram adicionados $50 \mu \mathrm{L}$ de ácido sulfúrico $2 \mathrm{M}$ em todos os orifícios para bloquear a reação. A leitura foi realizada em espectrofotômetro para microplacas de 96 poços utilizando-se filtro para comprimento de onda de $450 \mathrm{~nm}$.

Determinação das citocinas. A determinação dos níveis de IL-10 foi realizada através de ensaios imunoenzimáticos (ELISA), utilizando-se o soro sanguíneo estimulado com antígeno solúvel vacinal (VSA) e antígeno solúvel de L. chagasi (SLcA). Foram utilizados anticorpos e proteínas recombinantes R\&D Systems, Inc., Minneapolis, USA. Para determinar os níveis de IFN- $\gamma$, foi utilizado o kit DuoSets (R\&D Systems, Inc., Minneapolis, USA., DY1969, DY781) para realização do ensaio ELISA de captura em amostras de soro sanguíneo estimulado com antígeno vacinal e antígeno de L. chagasi. Os ensaios foram desenvolvidos de acordo com instruções do fabricante dos anticorpos R\&D Systems. A leitura foi realizada em espectrofotômetro para microplacas de 96 poços, Spectra Count TM (Packard Bio Science Company, EUA), utilizando-se filtro para comprimento de onda de $405 \mathrm{~nm}$.

Cálculo estatístico. A realização dos testes estatísticos foi com o apoio instrumental do software GraphPad Prism (Prism Software, Irvine, CA, USA). A normalidade dos dados foi demonstrada pelo teste $\mathrm{T}$ de Student. Os testes de análise de variância (ANOVA) seguido pelo teste de comparações múltiplas de Tukey foram empregados para avaliação entre os grupos do perfil de citocinas. Os dados obtidos foram considerados estatisticamente significativos quando o valor de $P<0,05$.

\section{RESULTADOS E DISCUSSÃO}

As formas promastigotas inoculadas na pele através do flebótomo são fagocitadas pelas células monocíticas podendo ser destruídas ou escapar da lise e proliferar em seu interior. Esta é a etapa onde ocorre a resposta imune do hospedeiro (Kontos \& Koutinas 1993).

Em nosso estudo a IL-10 parece ter suprimido a resposta imunológica protetora levando à progressão da doença com o aumento da expressão do parasito na célula. A alta produção de IL-10 observada, talvez indique que os macrófagos e as células T, dos cães infectados, estejam produzindo IL-10 em resposta ao antígeno de Leishmania e tal produção talvez seja importante para que o parasito persista nas células do hospedeiro.

Devido a inibição da função das células apresentadoras de antígeno, pela diminuição da expressão de moléculas do 
complexo MHC de classe II e moléculas coestimulatórias, impossibilitando as funções de apresentação de antígenos por células dendríticas e macrófagos.

Foi detectada a presença da citocina IL-10 no soro obtido dos dois grupos estudados (Fig.1) e, obtivemos diferenças estatísticas significantes $(P<0,05)$.

A alta produção de IL-10 pode estar relacionada a alta carga parasitária e o aumento da produção de anticorpos contribuindo para a patogênese da doença, causando danos teciduais pela deposição de imunocomplexos, que por sua vez podem estimular monócitos e macrófagos a produzirem mais IL-10 (Nylén \& Sacks 2007). No entanto, em trabalhos realizado por Quinnell et al. (2001), Santos-Gomes et al. (2002), Corrêa et al. (2006) e Manna et al. $(2006,2009)$ não foi constatado o papel imunossupressor da IL-10 na LVC. Verificou-se maior expressão de IFN- $\gamma$ nos cães infectados, sendo que, não foi observado aumento da expressão de IL-10 nestes animais, em relação aos animais não infectados.
O IFN- $\gamma$ é produzido por células T, dendríticas, macrófagos e células "natural killer" (NK) e tem a função de induzir a síntese de óxido nítrico, ativando funções microbicidas possuindo um espectro de ação muito amplo, sendo considerada uma das principais citocinas estimuladoras da imunidade contra infecções, atuando em ambas as respostas imunes, inata e adaptativa. É considerado o principal ativador de macrófagos, estimula a resposta imune por favorecer o crescimento de células Th1 em detrimento das Th2, e estimula a apresentação de antígenos pelo aumento da expressão de moléculas do MHC das classes I e II.

Foi detectada a presença da citocina IFN- $\gamma$ no soro obtido dos dois grupos estudados (Fig.2) e, obtivemos diferenças estatísticas significantes $(P<0,05)$. Observando um aumento significativo dessa expressão de citocina em animais assintomáticos, estimulando a apresentação de antígenos pelo aumento da expressão de moléculas do MHC das classes I e II e desencadeando resposta protetora à infecção, por meio da resposta Th1, Contrariando os achados de um

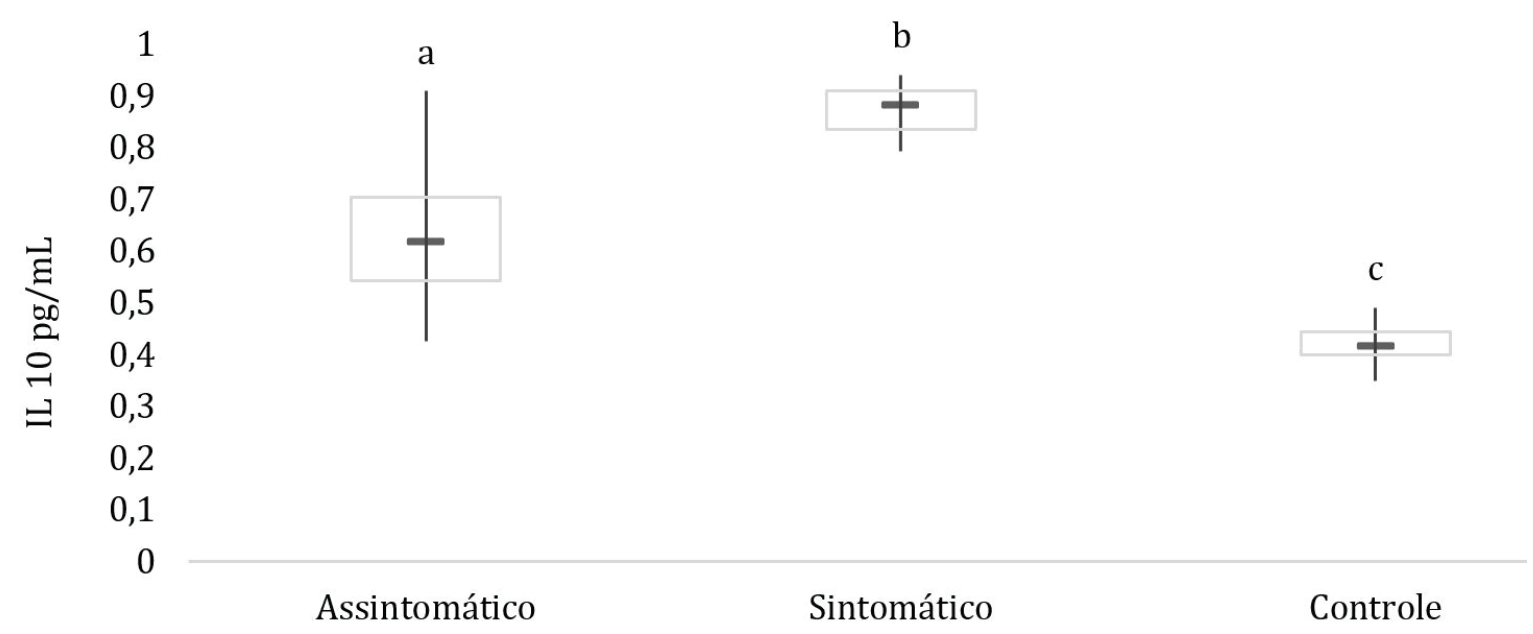

Fig.1. Quantificação de IL-10 no soro sanguíneo. Média e desvio-padrão do grupo de cães assintomáticos e sintomáticos, naturalmente infectados por Leishmania (L.) chagasi, obtidos no ensaio de ELISA de captura. As letras a, b e c mostram diferenças significativas $(P<0,05)$ em comparação com os grupos assintomático, sintomático e controle, respectivamente.

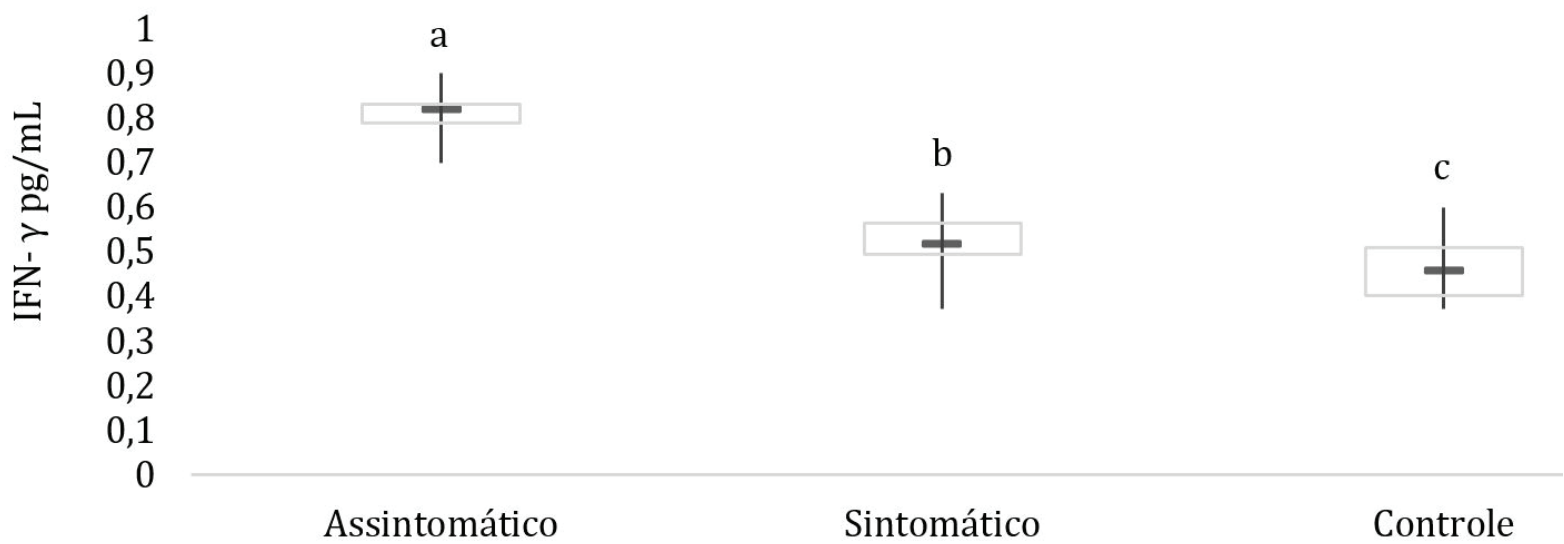

Fig.2. Quantificação de IFN- $\gamma$ no soro sanguíneo. Média e desvio-padrão do grupo de cães assintomáticos e sintomáticos, naturalmente infectados por Leishmania (L.) chagasi, obtidos no ensaio de ELISA de captura. As letras a, b e c mostram diferenças significativas $(P<0,05)$ em comparação com os grupos assintomático, sintomático e controle, respectivamente. 
trabalho desenvolvido por Sanchez-Robert et al. (2008) que foi demonstrado um aumento da expressão de IFN- $\gamma$ em CMSP nas formas clínicas mais graves da LVC, o que também aponta para o papel imunopatológico dessas citocinas nos processos relacionados a inflamação generalizada e visceral.

A incapacidade das células TCD4+ de secretar quantias significantes de citocinas ativadoras de macrófagos, tais como INF- $\gamma$, em resposta aos antígenos de Leishmania sp., tem importante papel na imunopatologia da enfermidade (Murray 1983, Carvalho et al. 1985).

Os fatores iniciais que contribuem para a inibição ou proliferação dos parasitos nas células do hospedeiro são pouco compreendidos, mas citocinas tais como IFN- $\gamma$ são conhecidas por influenciarem na sua replicação nos macrófagos (Murray et al. 1995, Wilson et al. 1998, Walker et al. 1999).

Os resultados provenientes da análise da expressão de IFN- $\gamma$, possibilitam especular que a expressão aumentada e simultânea desses mediadores deve estar intimamente envolvida com mecanismos imunoprotetores durante a LVC, visto que, observou-se aumento dessas citocinas no soro de animais portadores da forma clínica assintomática.

\section{CONCLUSÃO}

Esse estudo sugere que os perfis da expressão de citocinas são importantes para o controle da infecção e da manifestação dos sinais clínicos, pois contribuem para a compreensão dos mecanismos imunopatogênicos relacionados.

Agradecimentos.- À CAPES e à Fundação de Amparo à Pesquisa e Desenvolvimento Científico do Maranhão (FAPEMA) pelo apoio recebido.

\section{REFERÊNCIAS}

Carvalho E.M., Badaró R., Reed S.G., Jones T.C. \& Johnson Junior W.D. 1985. Absence of gamma interferon and interleukin production during active visceral leishmaniasis. J. Clin. Invest. 76(6):2066-2069. http://dx.doi. org/10.1172/JCI112209. PMid:3935667.

Corrêa A.P., Dossi A.C., Vasconcelos R.O., Munari D.P. \& Lima V.M. 2007. Evaluation of transformation growth factor $\beta 1$, interleukin-10, and interferon- $\gamma$ in male symptomatic and asymptomatic dogs naturally infected by Leishmania (Leishmania) chagasi. Vet. Parasitol. 143(3/4):267-274. http://dx.doi. org/10.1016/j.vetpar.2006.08.023. PMid:16979825.

Gontijo C.M.F. \& Melo M.N. 2004. Leishmaniose visceral no Brasil: quadro atual, desafios e perspectivas. Revta Bras. Epidemiol. 7(3):338-349. http:// dx.doi.org/10.1590/S1415-790X2004000300011.

Handman E. \& Bullen D.V. 2002. Interaction of Leishmania with the host macrophage. Trends Parasitol. 18(8):332-334. http://dx.doi.org/10.1016/ S1471-4922(02)02352-8. PMid:12377273.
Kontos V.J. \& Koutinas A.F. 1993. Old world canine leishmaniasis. Compend. Cont. Educ. Small Anim. 15(7):949-959.

Manna L., Reale S., Viola E., Vitale F., Manzillo V.F., Pavone L.M., Caracappa S. \& Gravino A.E. 2006. Leishmania DNA load and cytokines expression. levels in asymptomatic naturally infected dogs. Vet. Parasitol. 142(3/4):271280. http://dx.doi.org/10.1016/j.vetpar.2006.06.028. PMid:16920264.

Manna L., Reale S., Vitale F. \& Gravino A.E. 2009. Evidence for a relationship between Leishmania load and clinical manifestations. Res. Vet. Sci. 87(1):7678. http://dx.doi.org/10.1016/j.rvsc.2008.12.009. PMid:19178919.

Murray E.J. 1983. Beyond behavioural and dynamic therapy. Brit. J. Clin. Psychol. 22(Pt 2):127-128. http://dx.doi.org/10.1111/j.2044-8260.1983. tb00585.x. PMid:6871504.

Murray H.W., Hariprashad J., Aguero B., Arakawa T. \& Yeganegi H. 1995. Antimicrobial response of a $\mathrm{T}$ cell deficient host to cytokine therapy: effect of interferon-g in experimental visceral leishmaniasis in nude mice. J. Infect. Dis. 171(5):1309-1316. http://dx.doi.org/10.1093/infdis/171.5.1309. PMid:7751708.

Nylén S. \& Sacks D. 2007. Interleukin-10 and the pathogenesis of human visceral leishmaniasis. Trends Immunol. 28(9):378-384. http://dx.doi. org/10.1016/j.it.2007.07.004. PMid:17689290.

Paranhos M., Santos W.C., Sherlock I., Oliveira G.G. \& Carvalho L.C. 1993. Development of eosinophilia in dogs intradermically inoculated with sand fly saliva and Leishmania (Leishmania) chagasi stationary-phase promastigotes. Mem. Inst. Oswaldo Cruz 88(2):249-251. http://dx.doi. org/10.1590/S0074-02761993000200012. PMid:8107586.

Quinnell R.J., Courtenay O., Shaw M.A., Day M.J., Garcez L.M., Dye C. \& Kaye P.M. 2001. Tissue cytokine responses in canine visceral leishmaniasis. J. Infect. Dis. 183(9):1421-1424. http://dx.doi.org/10.1086/319869. PMid:11294678.

Sanchez-Robert E., Altet L., Alberola J., Rodriguez-Cortes A., Ojeda A., LópezFuertes L., Timón M., Sanchez A. \& Francino O. 2008. Longitudinal analysis of cytokine gene expression and parasite load in PBMC in Leishmania infantum experimentally infected dogs. Vet. Immunol. Immunopathol. 125(1/2):168-175. http://dx.doi.org/10.1016/j.vetimm.2008.04.010. PMid:18514330.

Santos-Gomes G.M., Rosa R., Leandro C., Cortes S., Romão P. \& Silveira H. 2002. Cytokine expression during the outcome of canine experimental infection by Leishmania infantum. Vet. Immunol. Immunopathol. 88(1/2):21-30 http://dx.doi.org/10.1016/S0165-2427(02)00134-4. PMid:12088641.

Walker P.S., Scharton-Kersten A.M., Krieg L., Love-Homan L., Rowton E.D., Udey M.C. \& Vogel J.C. 1999. Immunoestimulatory oligodeoxynucleotides promote protective immunity and provide systemic therapy for leishmaniasis via IL-12 and IFN- $\gamma$ dependent mechanisms. Proc. Natl Acad. Sci. USA 96(12):69706975. http://dx.doi.org/10.1073/pnas.96.12.6970. PMid:10359823.

Wilson M.E., Young B.M., Davidson B.L., Mente K.A. \& McGowan S.E. 1998. The importance of TGF-beta in murine visceral leishmaniasis. J. Immunol. 161(11):6148-6155. PMid:9834100. 